\title{
Carpal Coalitions; Failures of Differentiation of the Carpus: A Description of Cases
}

\author{
Anne J. Spaans, Annechien Beumer \\ Department of Orthopaedic Surgery, Amphia Hospital Breda, Breda, The Netherlands \\ Email: annespaans@gmail.com
}

Received January 30, 2013; revised February 26, 2013; accepted March 5, 2013

\begin{abstract}
Synostosis of carpal bones originates from lack of cavitation at the site of the future joint space with subsequent chondrification and ossification during the $4^{\text {th }}$ to $8^{\text {th }}$ weeks of intrauterine life. It is mostly seen as a chance finding discovered on radiographs. These coalitions are often asymptomatic, but can give complaints after trauma. We report six patients with different congenital carpal coalitions. These cases include synostoses of: scaphoid and trapezium, scaphoid and trapezium with a large scapholunate distance, lunate and triquetrum, capitate and trapezoid, capitate and hamate, triquetrum and pisiform. We also give a review of the literature and treatment proposal.
\end{abstract}

Keywords: Carpal; Coalition; Synostosis

\section{Introduction}

Synostosis of carpal bones is a congenital or acquired anomaly. Mostly, it is a chance finding discovered on radiographs obtained for other reasons [1]. These coalitions are often asymptomatic. However, they may give rise to complaints after trauma. Carpal synostoses occurs in about $0.1 \%$ of the population [2]. Congenital coalitions are considered to be a failure of differentiation. It may be transmitted as an autosomal dominant disorder $[1,3,4]$. We describe six cases of patients with different carpal synostoses as well as a review of the literature and treatment proposal.

\section{Case Reports}

\subsection{Case Report 1}

Patient A is a 21-year-old right-handed man of Dutch origin, who presented at the outpatient clinic with pain in the left wrist and an abnormal position and function of both elbows and wrists. He complained about inability to rotate the left forearm with a squeaking sensation and pain around the wrist. There was no trauma in the past. Examination of the wrist revealed a dorsal and palmar flexion of 70 degrees. Radial and ulnar deviation measured respectively 20 and 10 degrees with pain radial over the SL region. The same pain appeared with the Watson scaphoid shift test and to a lesser extent during the midcarpal shift test. Pro- and supination were both 45 degrees. Examination of the contralateral wrist demonstrated the same range of motion without pain. Radiographic examination of both hands showed bilateral a synostosis of scaphoid and trapezium. It also showed an ulna-plus configuration of $4.4 \mathrm{~mm}$ and a SL gap of $5.5 \mathrm{~mm}$ on the left side and an ulna-plus configuration of $3.0 \mathrm{~mm}$ and a SL gap of $4.0 \mathrm{~mm}$ on the right side (Figures 1-4). A radiograph of the left elbow showed a congenital dislocation of the radial head and a radiograph of the right elbow showed a mild dysplastic joint (Figures 5 and 6). Because of diminishing complaints, conservative treatment consisting of a removable wrist cast was advised, which was only used by this patient for a few weeks.

\subsection{Case Report 2}

Patient B (45-year-old) is the mother of Patient A. She presented at our outpatient clinic because of progressive pain in both wrists, mostly in the right wrist. Clinical examination showed pain with palpation at the location of the scaphoid, lunate and scaphoid-trapezium-trapezoid joint with a dorsal flexion of 70 degrees and palmar flexion of 15 degrees. Pro- and supination measured 30 respectively 80 degrees with a radial and ulnar deviation of both 10 degrees. X-rays and CT-scans of both hands showed bilateral a Madelung deformity with radiocarpal osteoarthritis. There was also a bilateral synostosis of the scaphoid and trapezium with a normal SL distance (Figures 7 and 8). In this patient, a wrist arthrodesis was performed at the right side at her special request.

\subsection{Case Report 3}

Patient C, an 18-year old woman, presented at our clinic 


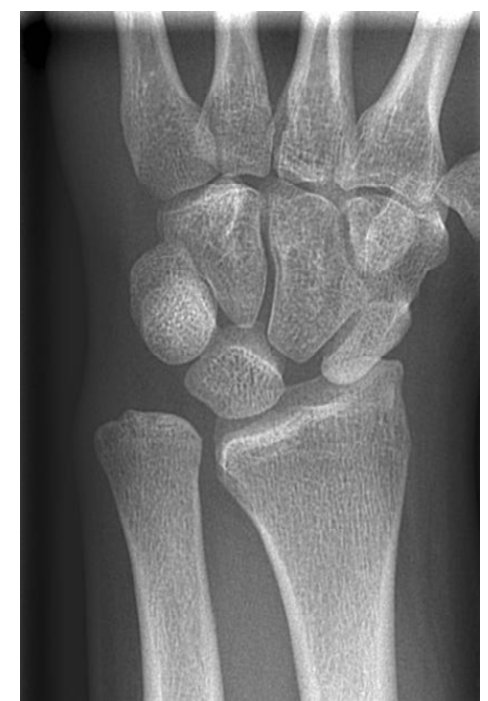

Figure 1. Left wrist of Patient A showing a synostosis between scaphoid and trapezium with SL gap.

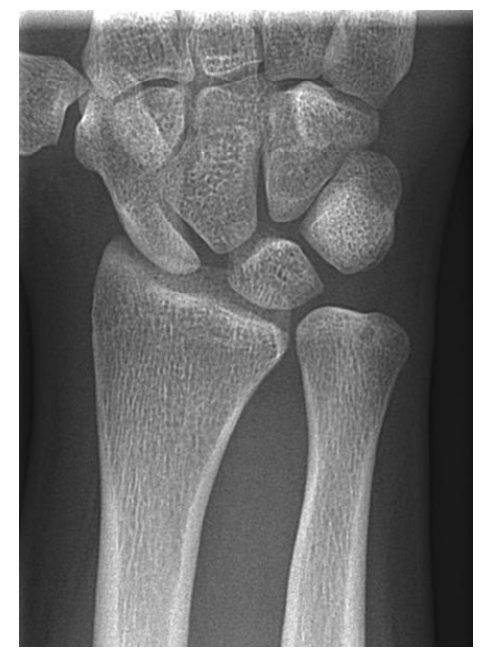

Figure 2. Right wrist of Patient A showing a synostosis between scaphoid and trapezium with SL gap.

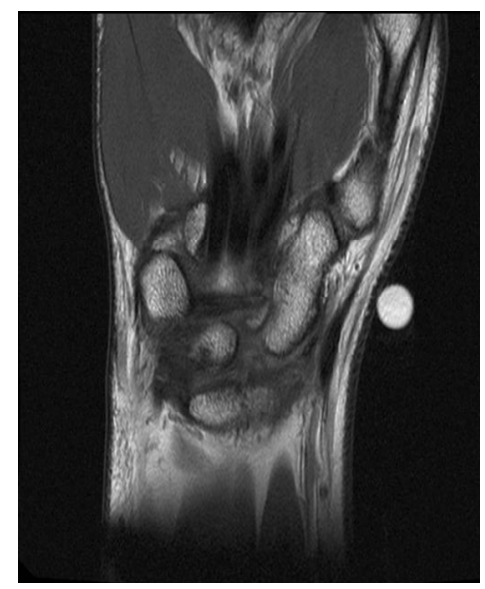

Figure 3. Left wrist of Patient A on MRI showing a synostosis between scaphoid and trapezium.

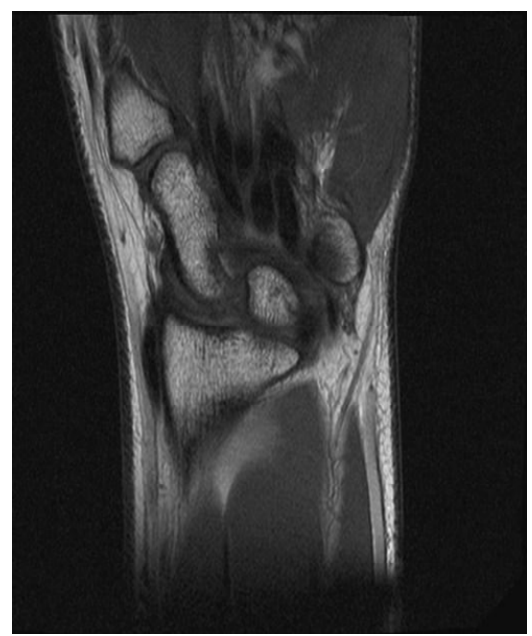

Figure 4. Right wrist of Patient A on MRI showing a synostosis between scaphoid and trapezium.

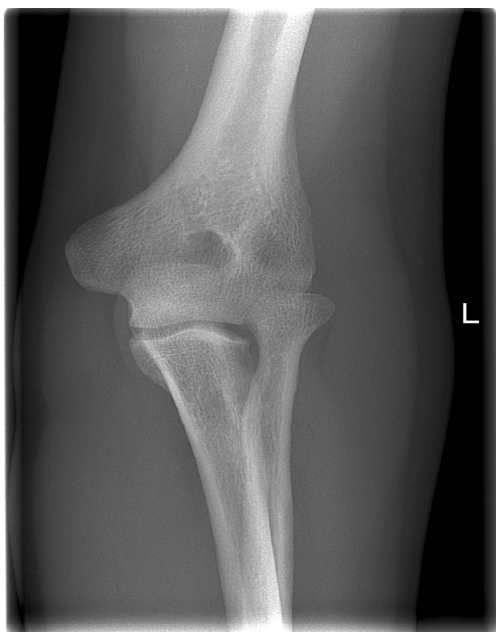

Figure 5. Left elbow of Patient A showing a congenital dislocation of the radial head.

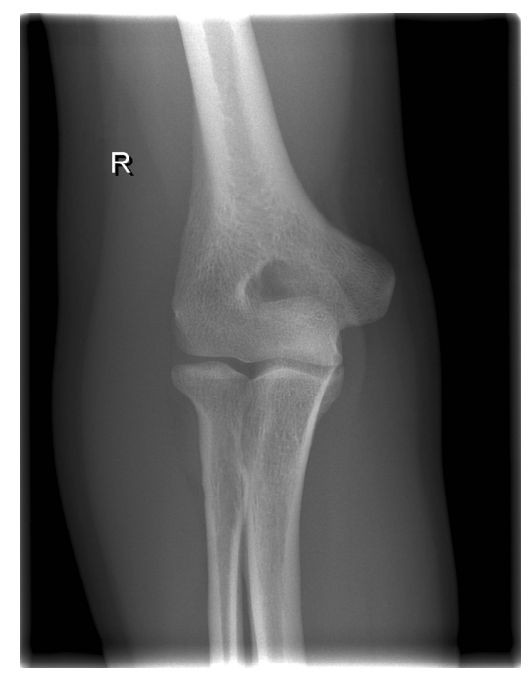

Figure 6. Right elbow of Patient A showing a mild dysplastic joint. 


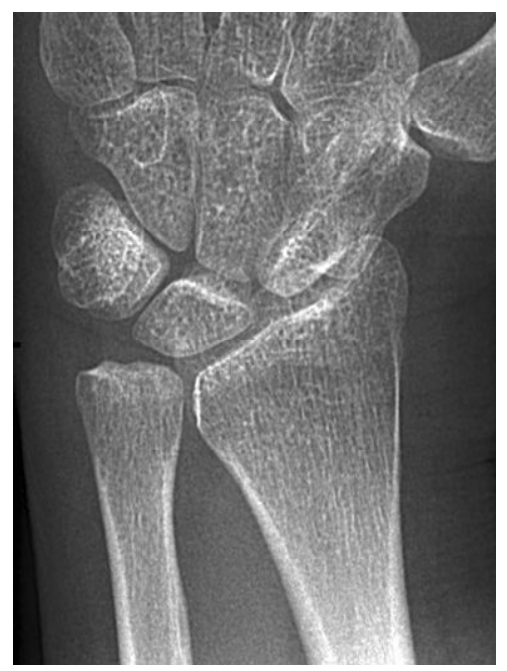

Figure 7. Left wrist of Patient B showing a synostosis of scaphoid and trapezium.

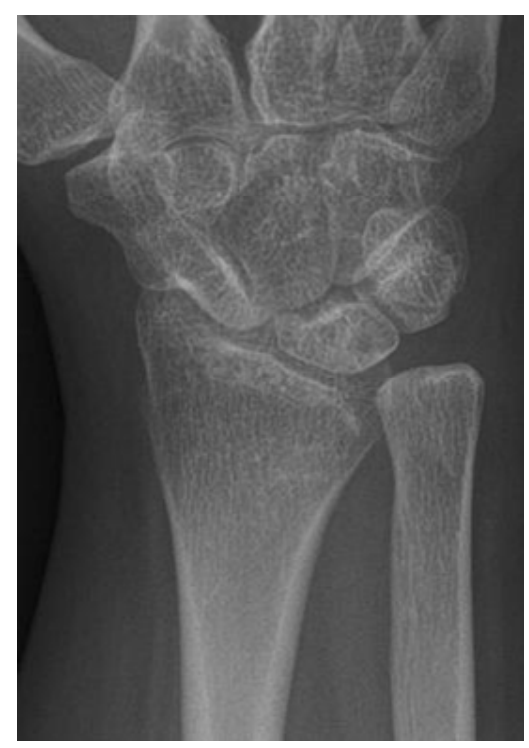

Figure 8. Right wrist of Patient B showing a synostosis of scaphoid and trapezium.

because of a painful click due to slight posterolateral rotational instability of the right forearm after a humerusfracture. There were no complaints of the wrist. Because of the rotational impairment, wrist X-rays were also taken. These showed a proximal radio-ulnar synostosis (Figure 9) and a coalition between lunate and triquetrum (Figures 10 and 11). Patient was advised to remove the intramedullary humerusnail. Because the carpal synostosis was asymptomatic, an expectative policy was followed.

\subsection{Case Report 4}

Patient D is a 38-year-old, otherwise healthy, woman with long lasting complaints of the left wrist, which in

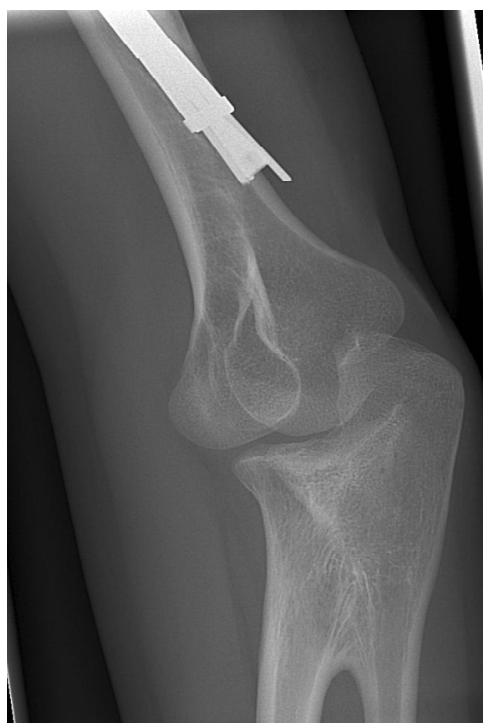

Figure 9. Right elbow of Patient $\mathrm{C}$ showing a proximal radioulnar synostosis.

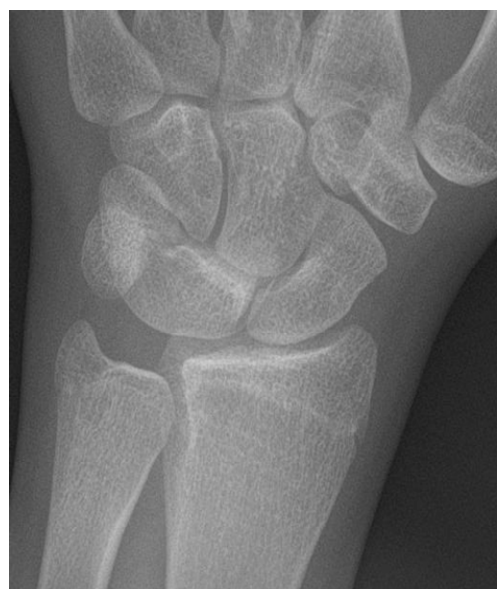

Figure 10. Left wrist of Patient $C$ showing a synostosis between lunate and triquetrum.

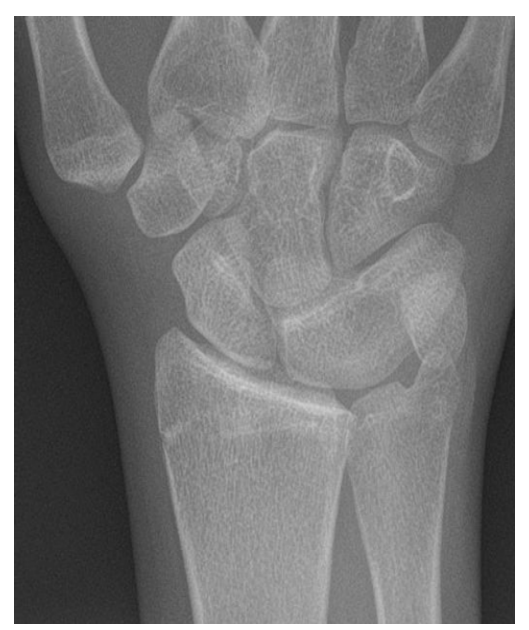

Figure 11. Right wrist of Patient $C$ showing a synostosis between lunate and triquetrum. 
crease during loading. There is also pain during driving and cycling. At clinical investigation there is pain over the thenar and the volar site of the distal forearm. Range of motion and stability are normal. Translation of the CMC 1 joint, pressure over the STT joint and the Watson test are painful without signs of instability. Midcarpal shift test is not painful. X-rays of the wrist show an irregular STT joint. An additional MRI shows a synostosis between capitate and trapezoid with slight bone marrow edema (Figure 12). A conservative policy was followed and patient was referred to a rehabilitation clinic.

\subsection{Case Report 5}

Patient E, an 18-year-old boy, presented at the emergency department after a fall of his bike with pain on both wrists. The right wrist showed some pain on the ulnar side of the carpus without swelling or limited range of motion. The left wrist showed swelling and pain over the scaphoid and distal radius with a limited range of motion. X-ray of both wrists showed no fractures, but a bilateral synostosis of capitate and hamate (Figures 13 and 14). At follow-up the complaints had disappeared and an expectative policy was followed.

\subsection{Case Report 6}

Patient $\mathrm{F}$ is a 41-year-old woman, who presented at our outpatient clinic because of pain in the right hand, most in the second, third and fourth finger. Clinical investigation showed no abnormalities. X-rays of the hand showed a coalition between hamate and capitate and a coalition between triquetrum and pisiform (Figures 15 and 16). An immobilising wrist splint was fitted.

\section{Discussion}

Congenital carpal coalition originate from lack of cavita-

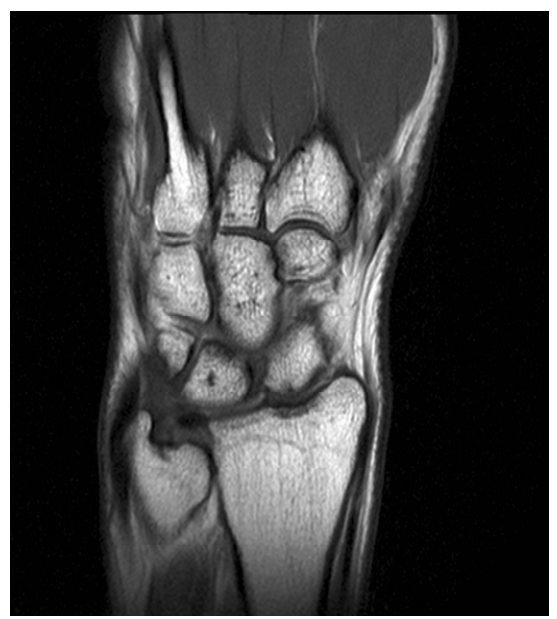

Figure 12. MRI of the left wrist of Patient $D$ showing a synostosis between capitate and trapezoid.

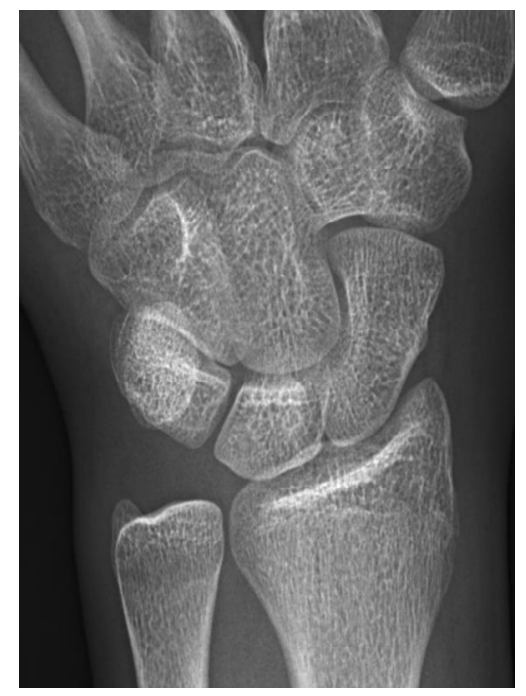

Figure 13. Left wrist of Patient $E$ showing a synostosis between capitate and hamate.

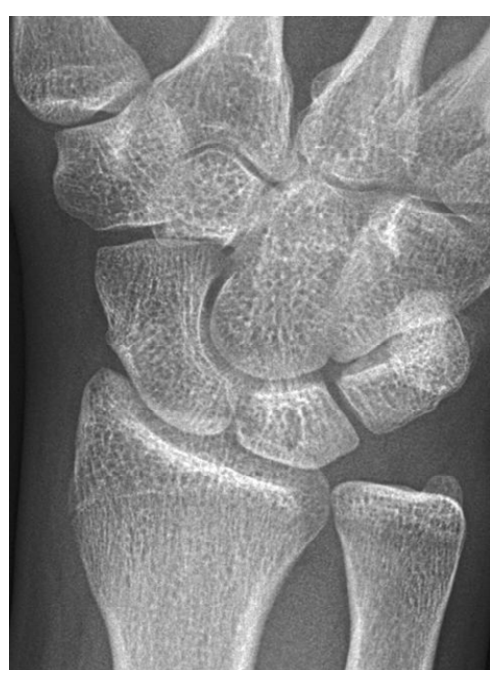

Figure 14. Right wrist of Patient E showing a synostosis between capitate and hamate.

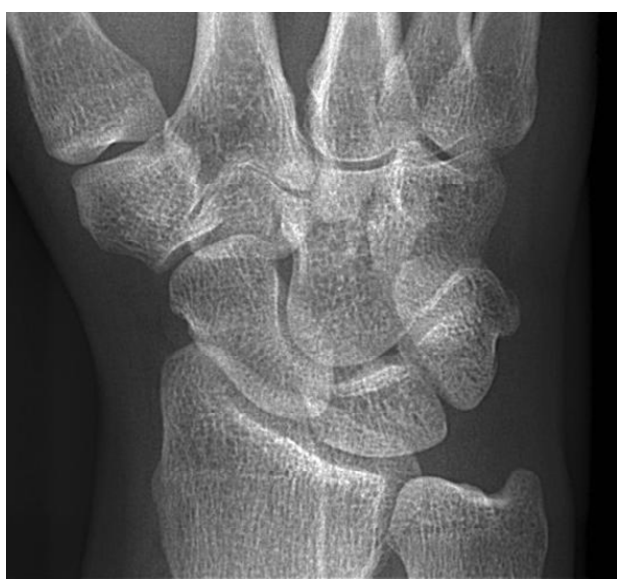

Figure 15. Right wrist of Patient $F$ showing a synostosis between hamate and capitate. 


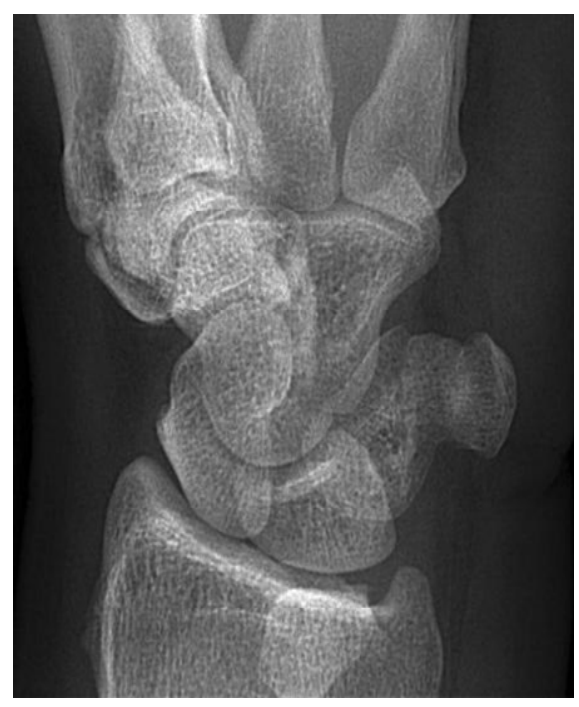

Figure 16. Right wrist of Patient $F$ showing a synostosis between triquetrum and pisiform.

tion at the site of the future joint space with subsequent chondrification and ossification during the $4^{\text {th }}$ to $8^{\text {th }}$ weeks of intrauterine life $[1,3,4]$. Almost every possible combination of congenital carpal synostosis has been reported [2-24]. The most common synostosis anomaly of the carpus is the lunate-triquetral coalition, followed by synostosis between the capitate and hamate $[4,15,16]$. For certain types of coalitions, there is a higher incidence amongst African people [1,3,19]. Synostoses can present as an isolated entity or can be part of a syndrome with multiple congenital anomalies, such as Steel syndrome, Muenke syndrome, congenital synspondylism, spondylocarpotarsal synostosis syndrome and arthrogryposis multiplex congenita [1]. Isolated synostoses tend to involve only two bones of the same carpal row. In contrast, syndromic carpal synostoses tend to involve more than two bones and cross the proximal and distal carpal rows [10, 22]. Synostosis of carpal bones can sometimes be hereditary and the trait is often transmitted as a Mandelian dominant factor, which is not sex linked [7].

Carpal synostosis normally does not interfere with the function of the wrist and is only rarely symptomatic [1]. However, some patients got pain and discomfort. After trauma, patients can complain about pain, a fracture through the synostosis, non-union after a fracture through the synostosis or a scaphoid luxation [13,18,24].

Minnaar de Villiers classified lunotriquetral coalitions into four radiological types (11, Table 1). This classification may be applicable to other carpal coalitions as well [21,22]. In Types 1 and 2 there can be some movement because of incomplete coalition, causing pain [14]. In Type 3, loss of movement between the fused carpal bones leads to a compensatory increase in motion at surrounding joints and can lead to stretching of the normal soft-tissue restraints, which can cause pain in stressful
Table 1. Classification of lunotriquetral coalitions according to Minnaar deVilliers [11].

\begin{tabular}{cc}
\hline Type 1 & $\begin{array}{c}\text { incomplete fusion resembling a pseudo-arthrosis } \\
\text { (fibro-cartilage coalition) }\end{array}$ \\
Type 2 & incomplete osseous fusion \\
Type 3 & complete osseous fusion \\
Type 4 & complete osseous fusion associated with other carpal \\
& anomalies \\
\hline
\end{tabular}

conditions [10].

In the present report different congenital carpal coalitions are mentioned in patients without known syndromes: synostosis of scaphoid-trapezium, lunate-triquetrum, triquetrum-pisiform, capitate-trapezoid and capitatehamate. One patient however had bilateral elbow problems. Other coalitions including nearly every possible combination of bones are described in the literature. There are coalitions of trapezium-trapezoid [20], scaphoidtrapeziumtrapezoid [22], scaphoid-trapezium-trapezoid-capitate [8], scaphoid-lunate-triquetrum [10] and pisiform-hamate [21, 24]. A widened scapholunate joint space, as seen in Case 1, had earlier been described by Metz et al. [12]. Although, all these patients had a congenital lunotriquetral coalition. It is important in patients with a coalition that cross the carpal rows to check if associated abnormalities are present because these synostosis are often part of a syndrome.

Treatment can be either conservative or operative, which depends on symptoms of the patient and the radiological type (incomplete, partial of complete coalition) [17,21]. Conservative treatment consists of elevation, relative rest or immobilisation, anti-inflammatory medication, cast immobilization and elimination of activities that precipitate pain $[9,10,14,17,23]$. If operative treatment is needed, a (partial) arthrodesis can be performed [22]. In tarsal coalitions, (arthroscopic) resection of the barr with or without interposition of material has been described [25,26]. In the wrist however, this has, to our knowledge, never been described.

\section{REFERENCES}

[1] P. Singh, A. Tuli, R. Choudhry and A. Mangal, "Intercarpal Fusion-A Review," Journal of the Anatomical Society of India, Vol. 52, No. 2, 2003, pp. 183-188.

[2] H. S. Hosalkar, B. A. Shaw, L. C. Carrie and H. Read, "Bilateral Congenital Capitate-Hamate Fusion," Images in radiology, Vol. 47, No. 3, 2001, pp. 208-209.

[3] T. J. Delaney and S. Eswar, “Carpal Coalitions,” Journal of Hand Surgery, Vol. 17, No. 1, 1992, pp. 28-31. doi:10.1016/0363-5023(92)90108-2

[4] R. O’Rahilly, "A Survey of Carpal and Tarsal Anomalies,” The Journal of Bone \& Joint Surgery, Vol. 35, No. 3, 1953, pp. 626-642. 
[5] T. Apard and F. Laumonier, "Congenital Scaphotrapezial Synostosis Associated with a Carpal Tunnel Syndrome: A Case Report,” Chirurgie de la Main, Vol. 28, No. 1, 2009, pp. 33-36. doi:10.1016/j.main.2008.11.008

[6] C. L. Barnes, G. T. Frazier and M. L. Hixson, "Bilateral Congenital Fusion of the Scaphoid and Trapezium in Identical Twins,” Orthopedics, Vol. 15, No. 6, 1992, pp. 739-741.

[7] M. B. Henry, "Anomalous Fusion of the Scaphoid and the Greater Multangular Bone,” Archieves of Surgery, Vol. 50, No. 5, 1945, pp. 240-241. doi:10.1001/archsurg.1945.01230030249004

[8] C. Ingram, R. F. Hall and M. Gonzal, "Congenital Fusion of the Scaphoid, Trapezium, Trapezoid and Capitate," Journal of Hand Surgery, Vol. 22, No. 2, 1997, pp. 167168. doi:10.1016/S0266-7681(97)80053-8

[9] K. Kaneko, K. Matsumara and Y. Maruyama, "Congenital Synostosis between the Scaphoid and the Trapezium as a Cause of Tenosynovitis Simulating de Quervain's Disease,” Chirurgie de la Main, Vol. 19, No. 3, 2000, pp. 187-190. doi:10.1016/S1297-3203(00)73478-0

[10] S. Knezevich and M. Gottesman, "Symptomatic Scapholunatotriquetral Carpal Coalition with Fusion of the Capitometacarpal Joint," Clinical Orthopedics, Vol. 251, 1990, pp. 153-156.

[11] A. B. Minnaar de Villiers, "Congenital Fusion of the Lunate and Triquetral Bones in the South African Bantu," The Journal of Bone \& Joint Surgery, Vol. 34B, No. 1, 1952, pp. 45-48.

[12] V. M. Metz, S. M. Schimmerl, L. A. Gilula, S. F. Viegas and P. Saffar, "Wide Scapholunate Joint Space in Lunotriquetral Coalition: A Normal Variant?” Radiology, Vol. 188, No. 2, 1993, pp. 557-559.

[13] H. Park and N. J. Goddard, "Re: Scaphoid Fracture in Bilateral Congenital Synostosis of the Scaphoid and Trapezium,” Journal of Hand Surgery, Vol. 34, No. 2, 2009, pp. 271-272.

[14] S. Peters and J. Colaris, "Carpal Coalition: Symptomatic Incomplete Bony Coalition of the Capitate and TrapezoidCase Report,” Journal of Hand Surgery, Vol. 36, No. 8, 2011, pp. 1313-1315. doi:10.1016/j.jhsa.2011.05.011

[15] C. Resnik, J. D. Grizzard, B. P. Simmons and I. Yaghmai, "Incomplete Carpal Coalition," American Journal of Roentgenology, Vol. 147, No. 2, 1986, pp. 301-304.

[16] B. Senecail, H. Perruez and D. Colin, "Numerical Variants and Congenital Fusions of Carpal Bones," Mor- phologie, Vol. 91, No. 292, 2007, pp. 2-13. doi:10.1016/j.morpho.2007.02.001

[17] E. Smith-Hoefer and R. M. Szabo, "Isolated Carpal Synchondrosis of the Scaphoid and Trapezium,” The Journal of Bone \& Joint Surgery, Vol. 67, No. 2, 1985, pp. 318320.

[18] M. P. Somford, M. F. A. M. Sturm and J. P. A. M. Vroemen, "Reconstruction of Isolated Scaphoid Dislocation with Carpal Dissociation, associated with a Carpal Anomaly," Strategies in Trauma and Limb Reconstruction, Vol. 5, No. 2, 2010, pp. 105-110. doi:10.1007/s11751-010-0092-X

[19] M. H. Sy, O. Diarra, M. Diagne, M. M. Diouf, P. Sene and S. Diouf, "Pyramido-Lunar Fusion in Black Africans. Apropos of 32 Cases,” Annales de Biologie (Paris), Vol. 39, No. 4-5, 1996, pp. 208-212.

[20] J. Terrence Jose Jerome, "Congenital Fusion of the Trapezium and Trapezoid," Romanian Journal of Morphology and Embryology, Vol. 49, No. 3, 2008, pp. 417-419.

[21] L. Tsionos, J. L. Drapé and D. Le Viet, "Bilateral Pisiform-Hamate Coalition Causing Carpal Tunnel Syndrome and Tendon Attrition. A Case Report,” Acta Orthopaedica Belgica, Vol. 70, No. 2, 2004, pp. 171-176.

[22] J. Weinzweig, H. Kirk Watson, T. J. Herbert and J. A. Shaer, "Congenital Synchondrosis of the ScaphotrapezioTrapezoidal Joint,” Journal of Hand Surgery, Vol. 22, No. 1, 1997, pp. 74-77. doi:10.1016/S0363-5023(05)80182-0

[23] S. M. Wilson, P. Moreel and E. Roulot, "Symptomatic Congenital Fusion of the Scaphoid and the Trapezium," Journal of Hand Surgery, Vol. 31, No. 5, 2006, p. 581. doi:10.1016/j.jhsb.2006.05.014

[24] P. H. Zeplin, R. G. Jakubietz and K. Schmidt, "Symptomatic Congenital Pisiform Hamate Coalition,” Annals of Plastic Surgery, Vol. 65, No. 4, 2010, pp. 396-397. doi:10.1097/SAP.0b013e3181d6e30e

[25] D. E. Bonasia, P. Phisitkul, C. L. Saltzman, A. Barg and A. Amendola, "Technical Note With Video Illustration. Arthroscopic Resection of Talocalcaneal Coalitions," Arthroscopy, Vol. 27, No. 3, 2011, pp. 430-435. doi:10.1016/j.arthro.2010.10.018

[26] A. L. Sarage, G. V. Gambardella, B. Fullem, A. Saxena and D. S. Caminear, "Cuboid-Navicular Tarsal Coalition: Report of a Small Case Series with Description of a Surgical Approach for Resection,” Journal of Foot and Ankle Surgery, Vol. 51, No. 6, 2012, pp. 783-786. doi:10.1053/j.jfas.2012.07.003 